\title{
Chatbot Introduction and Operation in Enterprises - A Design Science Research-based Structured Procedure Model for Chatbot Projects
}

\author{
Raphael Meyer von Wolff \\ University of Goettingen \\ r.meyervonwolff@uni-goettingen.de
}

\author{
Sebastian Hobert \\ University of Goettingen \\ shobert@uni-goettingen.de
}

\author{
Matthias Schumann \\ University of Goettingen \\ mschuma1@uni-goettingen.de
}

\begin{abstract}
Chatbot research has become an emerging research area. Researchers survey the technology behind and the whole ecosystem from different perspectives, e.g., human-computer interaction, design research, or anthropomorphism. To foster the transfer from research to practice, a comprehensive structured procedure model is missing yet. Due to this, the transfer of the research results into real-world settings in enterprises is often complicated. Hereto, we propose a comprehensive structured procedure model to guide practitioners in chatbot projects based on a Design Science Research study. In doing so, necessary project steps are pointed out and corresponding research results are highlighted to make them reusable for practice in a targeted manner. Thus, we provide structured support for chatbot projects in enterprises.
\end{abstract}

\section{Introduction}

Chatbot research is currently a widespread field of research in today's scientific community. Also from a practice perspective, the adoption of chatbots is increasing and more and more companies want to integrate chatbots into their application landscape. Due to digitalization efforts and intentions to support employees in enterprises more individually, chatbots or conversational agents are been applied in various scenarios like customer support, information acquisition, or e-learning [1-4]. Hereby, the research community adapts to this by surveying chatbots through different application areas [5-7] or by deriving design recommendations for creating chatbots $[7,8]$. Besides application area-centered research, researchers try to survey the chatbot ecosystem on a more general business level. Thereby, research directions tackle, e.g., trust aspects, humanizing the chatbot, and challenges [2, 9-12]. This generalized research can typically be reused and adapted for deviating scenarios or use cases. However, for productive applications of chatbots, e.g., in enterprises, these contributions only cover single aspects of the whole project lifecycle. Depending on project progress, different aspects need to be considered. The intertwining among the scientific findings is further difficult to trace and almost impossible to map through the individual contributions. Practitioners are faced with the challenge of implementing and carrying out their own chatbot projects due to missing guidelines. But also for science, the linkage of the contributions and their mutual effects cannot be made completely transparent. Thus, there is a need for a comprehensive structured framework on how to incorporate the existing research for conducting enterprise chatbot projects.

Up to now, some researchers already summarize their findings and deduce generalized design principles for enterprise chatbots [2, 13, 14]. However, only two approaches are known to us that aim at creating enterprise guidelines $[15,16]$. Hereby the first focuses only on the technical aspects while disregarding the organizational or individual ones. The second one addresses three partial aspects, i.e., use case, technology, and humanness, and derives best practices without integrating them into overall project organizations. Thus, comprehensive guidelines covering all relevant aspects of the chatbot lifecycle, i.e., technical, organizational, and individual, are missing. For applications in enterprises, the current research could often not easily be applied in a targeted manner. This can also hinder chatbot projects in businesses and result in lower outcomes, as critical aspects are forgotten, or wrong decisions are made.

Thus, our research aim is to construct a comprehensive business guideline for conducting chatbot projects. Therefore, we propose a procedure model for chatbot projects from an organizational-level, which (1) builds on previous experiences of chatbot projects, and (2) includes scientific results to guide future projects. Hereto, we conduct a Design Science Research (DSR) study $[17,18]$ and answer the research question:

\section{How to structure and conduct chatbot projects in} businesses based on actionable guidelines?

Next, we outline the related research. Then, we describe our DSR-approach. After this, we present our procedure model and discuss the results. 


\section{Related research}

Due to the increasing research in the last years, various terms emerged, e.g., chatbots [19], smart personal assistant [15], conversational agent [7], digital assistant [1], or conversational user interface [20]. However, all describe information systems that use artificial intelligence and machine learning in terms of natural language processing to provide a dialog-based user interface. Users can communicate naturally by voice or text to obtain information or perform functions. Technically, chatbots process natural language inputs to extract patterns and identify the users' intent. Based on the intent, the chatbot decides how to respond. Besides the chatbot's knowledge base, this requires integration with databases or (enterprise) systems [14].

With the use of chatbots in enterprises, e.g., for information acquisition, conducting business processes, or as a means for education, various potentials shall be achieved [5, 8, 14]. Among others, employees should be able to use systems without prior training due to their natural language interfaces [5, 19]; employees, especially in support areas, should be relieved through the chatbot by answering questions automatically, and, thus, processes become independent of further human resources $[7,14]$. So that all in all, systems become more user-centered and the quality of work is increased.

To reach these potentials of chatbots at workplaces, a lot of design research has been done so far. Besides the major focus on customer-focused areas, e.g., [7, 10, 21, 22], for example, Elshan and Ebel [23] survey chatbots as teammates, and Winkler et al. [6] apply them for problem-solving in businesses. Besides this, chatbots were also used as a means for feedback exchange [24]. For more process-like applications, Tavanapour et al. [25] support the ideation process with a chatbot. Hobert [26], and partly Chakrabarti and Luger [27], equip a chatbot with a finite state machine to dynamically map processes to support complex tasks and allow longer conversations. Additionally, Feine et al. [13], Diederich et al. [28], as well as Rietz et al. [29] summarize their findings by design principles for enterprise chatbots.

Since the research aim is to derive a comprehensive procedure model for practice, it is further necessary to identify meta-level research relevant on a project scale. As of now, a few studies can be found, that address this for enterprise applications of chatbots, e.g., in terms of application areas or use cases [8, 14]. Also, some approaches that focus on influencing factors and challenges during a chatbot project are available [11, 12]. Further studies bring together the existing scientific results in chatbot research in the form of literature reviews [2-4, 30-32] or to create a taxonomy of chatbots [33]. However, they categorize the results with a scientific focus and often do not provide guidance for practitioners. Additionally, Adam et al. [34] show that there are three HCI research modes, and what aspects are addressed and considered in each for respective projects A different approach highlights the knowledge needed for chatbot DSR-projects [35]. As mentioned in the introduction, Winkler et al. [15], and Schuetzler et al. [16] summarize scientific findings to apply them in a targeted process-oriented manner. However, only individual aspects are presented, so that usability for the entire chatbots' lifecycle is limited.

Thus, we build on previous research and create a structured guideline aligned with the lifecycle of chatbot projects. In doing so, we provide a meta-level DSR process-artifact to unite the previously achieved results to make them applicable in a targeted manner.

\section{Research design}

To unite the scientific results in chatbot research, and expertise from previous chatbot projects to deduce a comprehensive and generalized procedure model, we conducted a Design Science Research project according to Hevner et al. [17] and Hevner [18]. In doing so, we contribute with both (1) a problem-oriented process artifact, and (2) actionable guidelines to conduct chatbot projects in enterprises. Hereto, we conducted three iterations consisting of ten research steps (see Figure 1).

\section{1. $1^{\text {st }-I t e r a t i o n}$ - Constructing the artifact}

In the $1^{\text {st }}$-iteration of our DSR-project, we set up the initial process artifact (see Figure 1). (1) We examined the lack of missing enterprise support for structuring and carrying out chatbot projects in practice and derived the research problem. (2) Subsequently, we used existing scientific results as well as our own experience and expertise in chatbot research $[4,11,14,26,36]$ as the foundation. (3) Based on this, we deduced necessary steps and decisions to be taken in chatbot projects. Following a discussion in the research team, they were used to create the initial version of the procedure model. The initial model is logically aligned with classic software development processes, and consists of the phases planning, development, implementation, test, and operation, with a total of 21 steps (partial with substeps or selection options). See http://bit.ly/1st-Iter for the initial artifact. (4) Finishing the $1^{\text {st }}$-iteration, we evaluated the initial model. Hereto we conducted eight workshops with 10 local participants who conducted or guided chatbot projects on their own. The sessions lasted 66 minutes on average and were supported by an A0-print of the model. During the workshops, the model was discussed, and adjustments were recorded. 


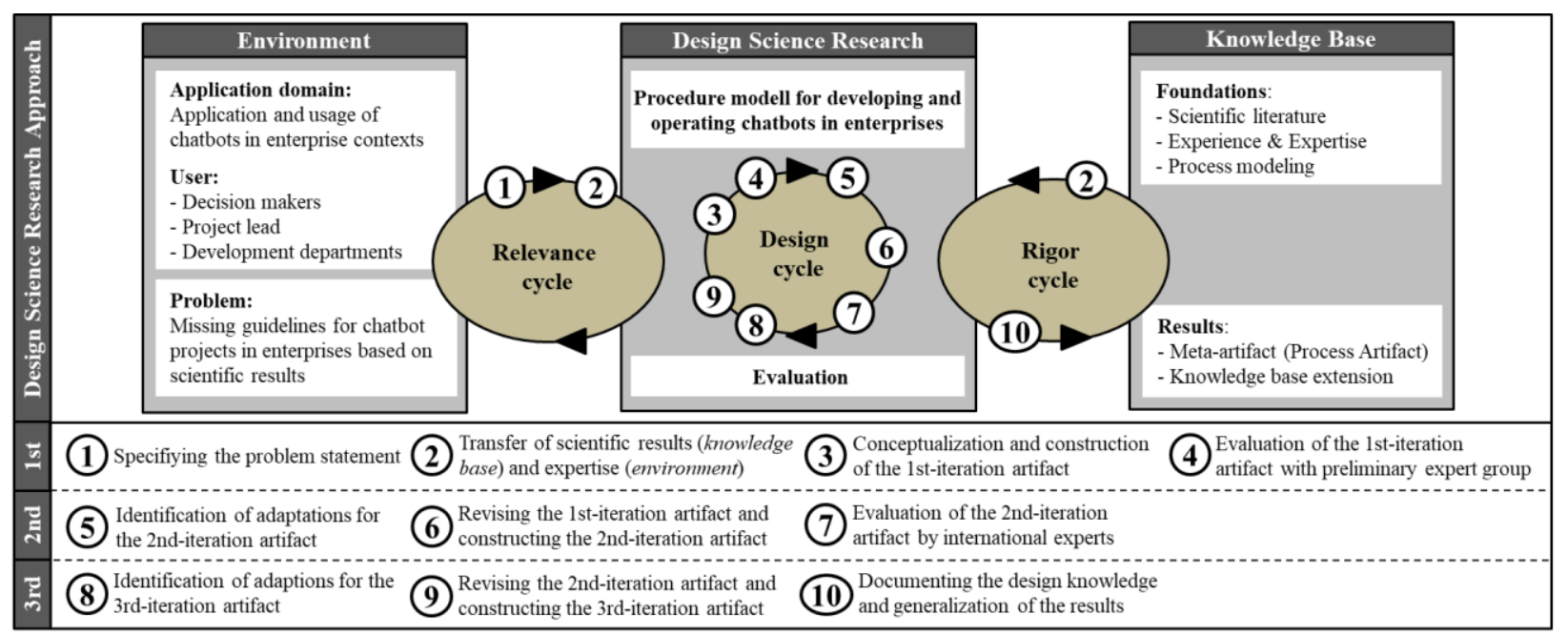

Figure 1. Applied design science research approach

\subsection{2nd-Iteration - Revising the artifact}

In the $2^{\text {nd }}$-iteration (see Figure 1), we (5) first summarized the evaluation results and derived enhancements. (6) Based on them, the initial model was adapted to develop our $2^{\text {nd }}$-iteration procedure model. Hereby, we extended and refined the initial procedure model by 15 steps, resulting in a 2nd-cycle procedure model consisting of 36 steps. In addition, returns and iterations are added to allow a more dynamic procedure model, and the phases were reduced to planning, development, test, and operation. See http://bit.ly/2nd-Iter for the $2^{\text {nd }}$-iteration procedure model. (7) The revised procedure model was evaluated to close the $2^{\text {nd }}-$ iteration. Hereto, we evaluated the procedure model on a broader and international scale with participants who (a) have experience in chatbot projects or develop chatbot projects, or are (b) involved in chatbot research to incorporate their scientific findings into our procedure mode. Therefore, we hosted a 2-hour workshop at last year's "CONVERSATIONS 2020"-conference [37]. This was attended by 13 international experts and researchers virtually due to the COVID19-situation. During the workshop and after initial brainstorming, each participant got access to the $2^{\text {nd }}-$ iteration procedure model via a website and could make annotations virtually. Lastly, our procedure model was discussed in the plenary, and notes were taken.

\subsection{3rd-Iteration - Finalizing the artifact}

Following, the $3^{\text {rd }}$-iteration started to develop our tentative final procedure model artifact (see Figure 1). Hereto, (8) the workshop findings, i. e., brainstorming results, discussion notes, and participants' annotations, were merged to identify enhancements. (9) Based on them, we created our tentative final $3^{\text {rd }}$-iteration procedure model for chatbot projects (see Section 4). Hereby, the $2^{\text {nd }}$-iteration procedure model was again refined and extended by five steps. (10) Lastly, we documented the findings in this contribution.

\section{A structured procedure model for chatbot projects in enterprises}

Next, our final procedure model for chatbot projects is described (see Figure 2-5; the entire image is available at: http://bit.ly/CB PM). Thereby, chatbot projects should encompass the phases: planning, development, test, and operation, which are passed through one after the other while allowing returns or iterations.

\subsection{Planning phase}

Typically, chatbot projects begin with the planning phase. Hereby, general conditions and objectives need to be defined like in any typical software-related project. Also, organizational, technical, and individual foundations and capacities are created in the firm and the overall project is set (see Figure 2; enhancements between $1^{\text {st }}$ and $3^{\text {rd }}$-iteration are highlighted by color).

According to our findings, a chatbot project should start 1 (see Figure 2) with the fundamental plan to introduce and operate chatbots in an enterprise situation. If this, was mainly driven by the customers or users, the enterprise has to survey the user's persona in order to define them appropriately 1.1. Based on this, customers or users' requirements for the chatbot solution can be deduced by analyzing them 1.2 . If the initiative was started based on the enterprise or stakeholders, the company itself, targeted processes, and application areas must be analyzed 1.3 . Depending 


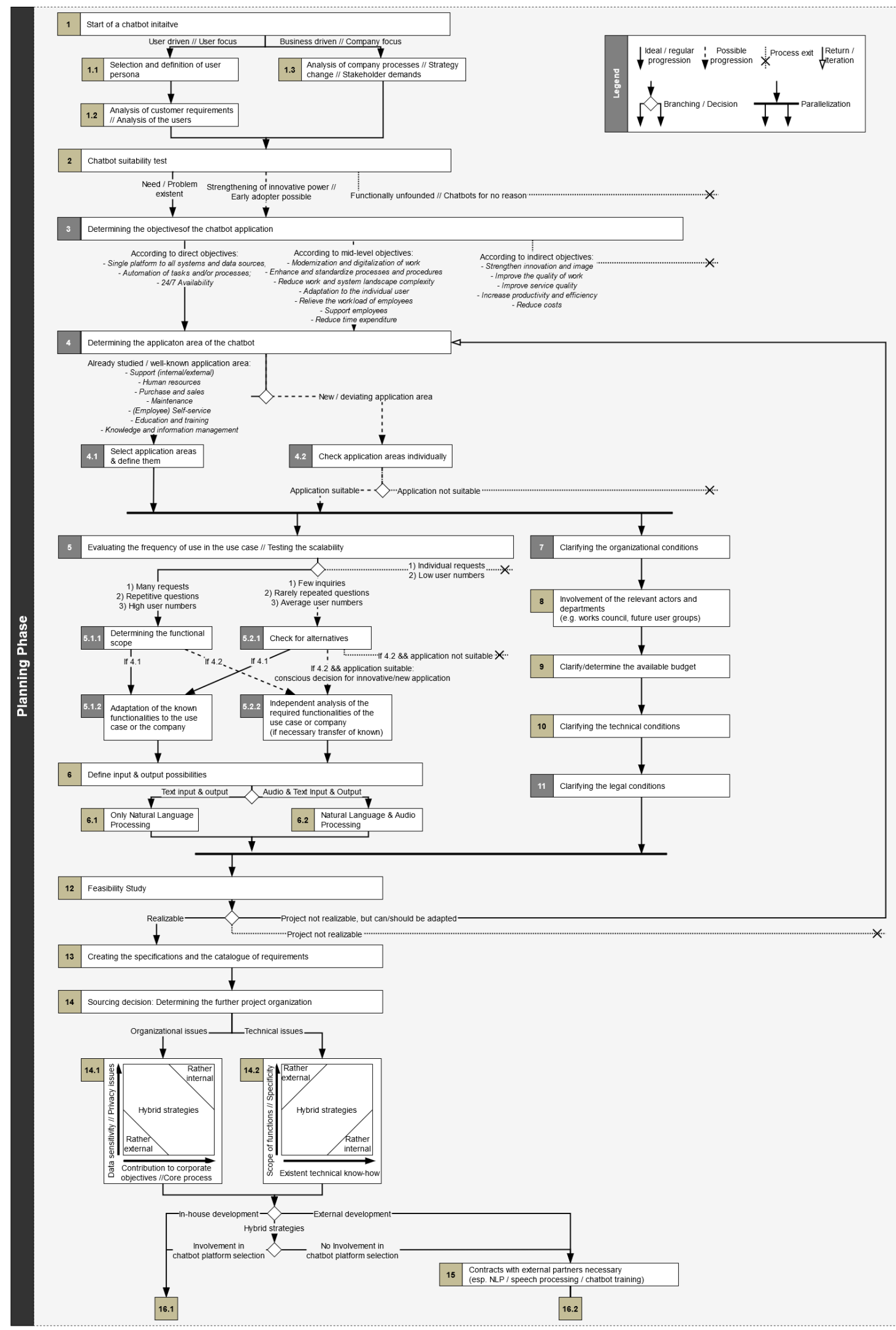

Figure 2. Planning phase 
on the results, a first suitability test, if a chatbot is the right solution, should be conducted 2 [16]. It is advisable to pursue the project only if there is a real need or if a problem can be solved by using a chatbot. Under certain circumstances, it is also advisable to use a chatbot, e.g., to emphasize the company's innovative strength or to set itself apart from competitors as an early adopter. If the chatbot is functionally unfounded or the initiative was started due to the technological hype around chatbots $[12,19]$, it should be considered to stop the project as early as possible. Further, 3 companies should be clear about the specific goals they actually want to pursue with the project and whether these can be met with a chatbot, e.g., those of [14, 38]. Only if the objectives can be addressed directly, the project should be pursued further. Otherwise, the question arises whether chatbots are really the solution since the objectives can only be addressed indirectly and are not the immediate focus of chatbots. Following this, 4 the application area must be determined. Hereby, already established application areas are especially suitable for an enterprise application. As (a) many research results are already existent and (b) previous generalized design recommendations could be reused. Overviews of possible chatbot application areas can be found for example in $[3,8,14]$. If a deviating application area is selected, an individual review is necessary, based on which the project can be followed or stopped. As chatbots are especially useful in scenarios, where many requests, repetitive questions, or high user numbers are existent, the potential usage frequency and scalability must be evaluated 5 . If the conditions are given, the functional scope is to be defined. Otherwise, it should be reflected whether chatbots are really the solution for the problem/need. Depending on the selected use case, existent results as highlighted in 4 can be transferred and applied, or an independent analysis is necessary. Also, 6 in- and output setting should already be determined in the planning phase since this influences the functional scope. Typically, a choice can be made between text/audio-only or audio and text in- \& output, e.g., [31]. In parallel, the organizational conditions must be clarified 7 , relevant actors, e.g., works council or future users, should be involved and the project team for the further course should be created 8 . In addition, 9 the budget planning must be carried out to determine the available budget. Also, technical conditions 10 , e.g., infrastructure and IT-knowhow, and legal conditions 11 , e.g., data security and protection or processing of language/personal data, have to be clarified. By bringing the results of 5 to 11 together, a feasibility study can be performed 12 , which marks the conscious choice for continuing the chatbot project and, thus, can be seen as a first milestone. However, if the basic decisions are not feasible, consideration can also be given to adaptation. After this feasibility study, the specifications and the requirements catalog can be derived and assembled 13. This should be as comprehensive as possible at that point, but should also be continuously monitored and adapted during the further course of the project. The current research can provide starting points for requirements in terms of design principles, e.g., [7, $13,21-23,28]$. Next, the sourcing decision is necessary 14. Typically, the choice is between in-house, external/outsourcing, or hybrid approaches. However, for external constellations contracts are further necessary, e.g., for data/NLP processing 15 . Based on our workshops, possible portfolios are 14.1,14.2 (a) organizational: fit between data sensitivity/privacy and contribution to the corporate objectives/processes, and (b) technical: fit between functional scope/specificity and the existence of technical know-how in the firm. Depending on the selection, the development can start.

\subsection{Development phase}

Following the planning, the development starts where the chatbot will be set up according to the requirements of a selected use case. Hereto, mostly technical tasks are necessary to further refine the requirements and implement the chatbot (see Figure 3; differences between $1^{\text {st }}$ and $3^{\text {rd }}$-iteration colored).

Depending on the sourcing decision, the phase starts with the selection of a chatbot platform or framework 16 [15]. Under certain circumstances, the choice might be dependent on the external partner selected for outsourcing. As many different solutions exist, e.g., RASA, Google Dialogflow, IBM Watson, or NLP.js, some existing overviews, to begin with, can be found for example in [16, 22, 39]. Furthermore, an appropriate database architecture must be defined 17, if not determined by the platform/framework. Subsequently, the integration with existing enterprise systems must be determined 18 . Hereby, if integration is wanted, the desired available enterprise systems or databases must be analyzed in terms of their interface capabilities 18.1 and the integration should be performed by using existing or newly developed interfaces 18.2 . Otherwise, also a stand-alone chatbot operation is possible 18.3 . If the desired integration is not possible, the only option is to consider a change of architecture or an adaptation of the requirements 18.4. Besides the integration with enterprise systems, a user interface or rather a channel integration is also necessary 19 . Hereto, the chatbot must be integrated into the desired end devices or UIs or made be available as a new channel, e.g., for customer support. Also, the chatbots' 


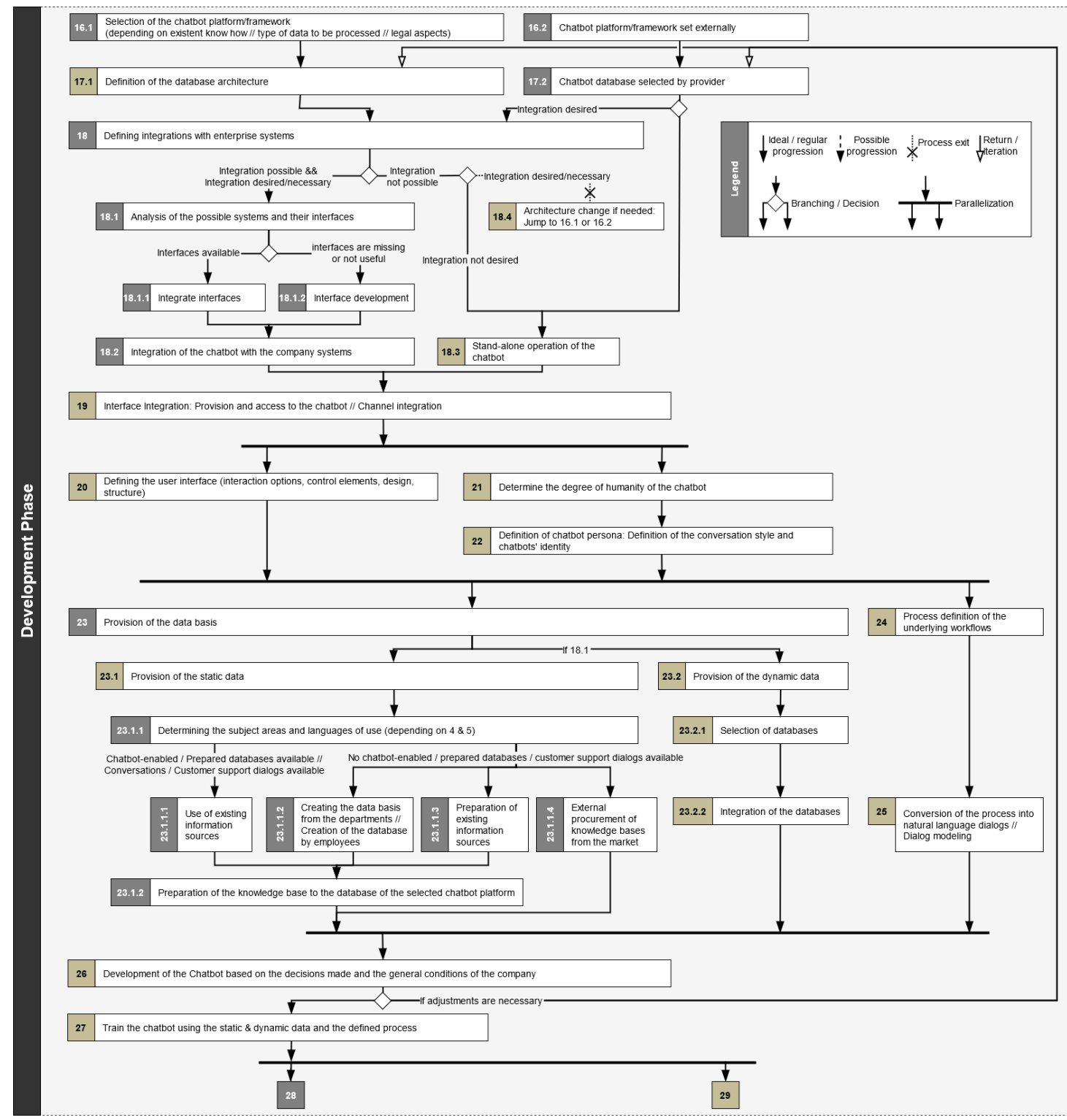

Figure 3. Development phase

user interface must be defined 20 , e.g., control options and elements, design and colors, or overarching UI structure. In addition, our workshop participants pointed out, that in this step also the desired level of humanity and anthropomorphism must be clarified 21. Hereto, enterprises can already rely on a large research stream, e.g., $[9,10,28,29,40]$. This also encompasses the definition of chatbots' persona 22 [16], e.g., conversation style, appearance, or name. Following this, the critical task of providing the chatbots knowledge starts. First, the knowledge/data must be provided 23, which encompasses both the static data and the dynamic data. For the former 23.1, and taking into account the application area and functionalities as well as the target language, four possible options are existent according to our workshops: (a) if chatbot-capable databases or documented user dialogs are available, the existing sources should be used directly. If none of these prerequisites exist, (b) the necessary data must be created in-house, e.g., by the customer department, (c) existing sources must be prepared in such a way that they are usable, or (d), if available, knowledge could be procured externally. For the latter 23.2, the available data sources must be selected and integrated. Second, if the chatbot should encompass or map a (business) process, the respective one must be defined 24 and transferred to natural language dialog form in terms of dialog modeling 25 [15]. Finally, the chatbot can be developed depending on the expertise and desired development approach of the respective company, e.g., 

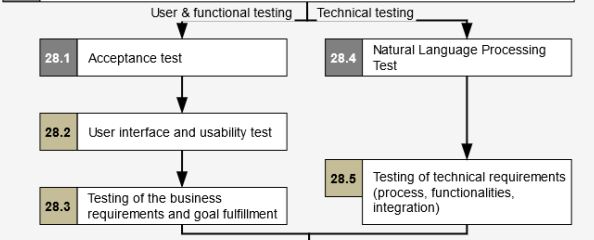

Figure 4. Test phase

using SCRUM 26. Also, the initial chatbot training using the defined and provided static and dynamic data as well as the targeted (business) process happens 27 .

\subsection{Test phase}

Next, testing begins where the chatbot is reviewed from the users' and technical perspectives (see Figure 4; enhancements between $1^{\text {st }}$ and $3^{\text {rd }}$-iteration are colored).

Hereto, the chatbot testing 28 encompasses both user and functional tests, as well as technical tests [15]. For the former, the acceptance for the system among future users should be determined 28.1. In addition, the user interface and the resulting users' experience should be subjected to testing 28.2. Hereto, previous studies already applied common metrics, like the user experience questionnaire, chatbot usability questionnaire, or the system usability scale, like in [20, 41]. Some reviews for possible chatbot evaluations are already existent [36, 42]. Also, the fulfillment of the objectives and business requirements must be checked 28.3. From a technical perspective, the natural language processing capabilities 28.4 and the technical requirements 28.5 must be assessed to identify adjustments. In addition, it was emphasized during the workshops that access to the chatbot should be made available as early as possible for selected user groups so that their feedback can be considered as soon as possible 29. Hereby, an indication notice about the current state of development is necessary, e.g., alpha or beta version. By bringing these test and assessment results together 30 , it can be checked, if the current instantiation complies with the specifications and can be released for productive operation. Depending on the results, the next phase starts or the chatbot must be revised.

\subsection{Operation phase}

After testing, the chatbot operation begins. Hereto, organizational measures must be taken to successfully operate the chatbot, and continuous technical adaption is necessary to ensure error-free operation (see Figure 5; enhancements between $1^{\text {st }}$ and $3^{\text {rd }}$-iteration are colored).

After the "Go Live" 31, on the one hand, organizational issues must be taken into account. Especially, a change management is necessary 32 to promote awareness for the new system and to demonstrate and show the added value generated by the new chatbot system. In addition, training should be offered to future users to get to know the system and how to use it. This can also be done by HowTo's or help pages in the system. Further, the existing channels and the chatbot's capabilities should be compared. At least, during the transition, they should be maintained in parallel 33 . If the chatbot replaces existing channels, enterprises should consider switching off the alternative channels 33.1 to free up resources that can be used elsewhere 33.2. Otherwise, permanent operation of the chatbot and the other channels seems the only viable option 33.3. In addition, enterprises must build up organizational structures for chatbots' maintenance, while also appoint someone who is responsible 34 . The responsible team should also go through training for chatbot care and maintenance. From the technical perspective, it is necessary to continuously care and maintain the chatbot 37 [15]. Hereto, the chatbot usage and the chatbot dialogs have to be evaluated regularly 38. Based on these evaluations, which can also include aspects from the test phase, existing problem sources or errors are to be identified 39. According to them, it could be necessary to (a) retrain the NLP algorithm for a better speech understanding, (b) adjust the knowledge base or the underlying process, so that the chatbot learns the missing answers or perform activities, or (c) update the chatbot from a technical perspective to fix bugs and errors. Regardless of the case, this entire process, starting at 38 , must be carried out regularly and, above all, promptly 40 . Otherwise, there is a risk that users will quickly stop using the chatbot because it does not help them, or functionalities are not carried out, e.g., due 


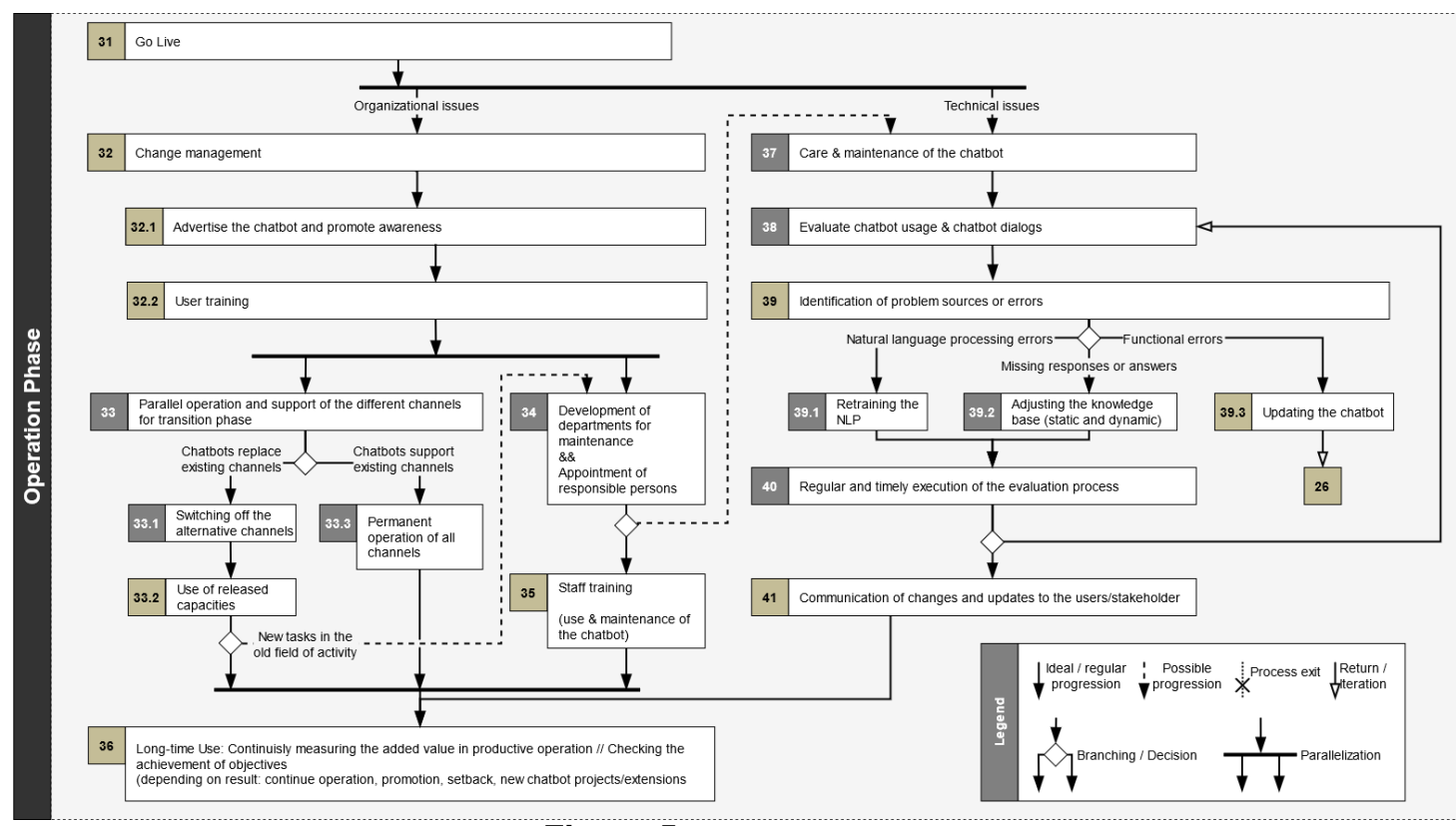

Figure 5. Operation phase

to a lack of natural language understanding. In addition, adjustments and updates made should be communicated with the users to make improvements visible. For the last step, our workshop participants noted that the chatbot operation must be measured and evaluated from a long-term perspective 36. Hereby, the real added value can be identified and the achievement of the initial objectives 1-3 can be measured. Depending on this, continuous operation is possible. However, also further promotion of the chatbot could be necessary, as well as setbacks or necessary adoptions up to a stop of the project. Nonetheless, also new chatbot projects can be identified as well as possible extensions to the current.

\section{Discussion and conclusion}

Based on a Design Science Research approach [17, 18], we surveyed the applicability of scientific results and developed a comprehensive structured procedure model as a guideline for chatbot projects in enterprises.

First, we show that for many individual tasks regarding chatbot projects existing research can be applied. However, this research mostly addresses design research and corresponding requirements, their evaluations, as well as studies for anthropomorphism. To make those research results applicable in practice, practice-focused approaches are needed. Especially chatbot project-related research is missing, e.g., project organization, sourcing, operation, etc. Nonetheless, comprehensive reviews are existent on which one can build as a starting point, e.g., [2-4, 30, 32].

Secondly, we show that chatbot projects should be aligned alongside the four phases planning, developing, testing, and operating while performing up to 41 tasks (see Figure 2-5). To allow flexibility and dynamic also some iterations or step-backs are necessary, e.g., if adjustments must be made. By evaluating the procedure model twice, the findings could be verified. Hereby, we could show that the prior process-oriented research approaches [15, 16], cover important aspects within projects, but we could incorporate them into a comprehensive procedure model that now maps the entire chatbots' lifecycle. However, we propose a sequential order, due to better readability and simplification of the presentation in this contribution. Nevertheless, this does not mean that the order is rigid. Rather, it is also possible to deviate from the sequence or to have several tasks in parallel, than shown by us. In doing so, each willing company can use and adapt the model depending on its own characteristics and resources. However, it is important that at the end of a phase all steps of the phase have been completed and that a result has been determined for each of them. Otherwise, steps might be forgotten or decisions are made that cannot be reversed later. In this project, we deliberately did not define the actual development. This makes the resulting procedure model independent of future technological improvements. Furthermore, depending on the enterprise or the development experience, e.g., SCRUM or other forms of development procedure may be conceivable here. Hence, typical software development procedures can be applied and the respective enterprise can choose where they themselves have the most knowledge. Necessary training or learning of new procedures can be avoided. Nevertheless, critical concerns can be raised about the 
necessity of a specific procedure model for developing enterprise chatbots. We argue that chatbots differ substantially from classic systems, especially through the use of AI and NLP. There are new steps that do not have to be taken into account in classic IT-systems, e.g., continuous training or knowledge provision. Especially, here a linkage of existing research seems essential, as chatbots are still an emerging technology. Therefore, our procedure model is a design contribution in terms of a process artifact on how to transfer established chatbot knowledge into a company for practical usage.

Despite our results, there exist some limitations to be noted. First, our results rely mostly on scientific research and own chatbot experience. However, as we evaluated and enhanced our model twice, we expect it to be of high accuracy. In particular, the number of changes decreased between the iterations. Second, since the study mainly involved researchers, it is possible that practice-related aspects were overlooked or given less consideration. Thus, to measure the actual practicality, our proposed procedure model should be used for actual enterprise chatbot projects as a guideline. Thereby, a case study can be conducted to generalize our findings.

In conclusion, we contribute to both, practice and research. Chatbot project managers can utilize the results to plan chatbot projects and ensure that no steps are forgotten and important decisions are made. The project can be easier communicated to stakeholders, management, or inside the team/department. Also, as we align corresponding research, they are easier to apply and the existing research can be considered in a targeted manner. Chabot programmers gain insight into design recommendations and configurations and can actively incorporate them into their developments. They can also more easily plan and finalize their development steps. For chatbot researchers, we could provide a basis for future chatbot studies and show an approach to make chatbot-related research usable. Further, we highlight topics that are relevant for enterprise chatbot research.

\section{Acknowledgement}

We thank the organizers of "CONVERSATIONS 2020" [37] for the opportunity to host a workshop and the workshop participants for their helpful feedback.

\section{References}

[1] A. Maedche, C. Legner, A. Benlian, B. Berger, H. Gimpel, T. Hess, O. Hinz, S. Morana, and M. Söllner, "AI-Based Digital Assistants: Opportunities, Threats, and Research Perspektives", Business \& Information Systems Engineering 61(4), 2019, pp. 535-544.

[2] S. Diederich, A.B. Brendel, S. Morana, and L.M. Kolbe, "On the Design of and Interaction with Conversational
Agents: An Organizing and Assessing Review of Human-Computer Interaction Research", Journal of the Association for Information Systems (Forthcoming), 2022, pp. 1-69.

[3] S. Feng and P. Buxmann, "My Virtual Colleague: A Stateof-the-Art Analysis of Conversational Agents for the Workplace", 53rd Hawaii International Conference on System Sciences, Maui, Hawaii, 2020, pp. 156-165.

[4] R. Meyer von Wolff, S. Hobert, and M. Schumann, "How May I Help You? - State of the Art and Open Research Questions for Chatbots at the Digital Workplace", 52nd Hawaii International Conference on System Science, Maui, Hawaii, 2019, pp. 95-104.

[5] S. Carayannopoulos, "Using chatbots to aid transition", International Journal of Information and Learning Technology 35(2), 2018, pp. 118-129.

[6] R. Winkler, M.L. Neuweiler, E. Bittner, and M. Söllner, "Hey Alexa, Please Help Us Solve This Problem! How Interactions with Smart Personal Assistants Improve Group Performance", 40th International Conference on Information Systems, Munich, Germany, 2019, pp. 1-17.

[7] U. Gnewuch, S. Morana, and A. Maedche, "Towards Designing Cooperative and Social Conversational Agents for Customer Service", 38th International Conference on Information Systems, South Korea, 2017, pp. 1-13.

[8] S. Laumer, F. Gubler, A. Racheva, and C. Maier, "Use Cases for Conversational Agents: An Interview-based Study", 25th Americas Conference on Information Systems, Cancun, Mexico, 2019, pp. 1-10.

[9] A.-M. Seeger, J. Pfeifer, and A. Heinzl, "When Do We Need a Human? Anthropomorphic Design and Trustworthiness of Conversational Agents", SIGHCI 2017 Proceedings, 2017, pp. 1-6.

[10] C. Liebrecht and van Hooijdonk, "Creating Humanlike Chatbots: What Chatbot Developers Could Learn From Webcare Employees In Adopting A Conversational Human Voice", in Chatbot Research and Design: CONVERSATIONS 2019, A. Følstad, et al., Eds., 2020, Springer International Publishing: Cham, pp. 51-64.

[11] R. Meyer von Wolff, S. Hobert, and M. Schumann, "Sorry, I Can't Understand You! - Influencing Factors and Challenges of Chatbots at Digital Workplaces", 16th International Conference on Wirtschaftsinformatik, Essen, Germany, 2021, pp. 1-16.

[12] D. Rodríguez Cardona, O. Werth, S. Schönborn, and M.H. Breitner, "A Mixed Methods Analysis of the Adoption and Diffusion of Chatbot Technology in the German Insurance Sector", 25th Americas Conference on Information Systems, Cancun, Mexico, 2019, pp. 1-10.

[13] J. Feine, M. Adam, I. Benke, A. Maedche, and A. Benlian, "Exploring Design Principles for Enterprise Chatbots: An Analytic Hierarchy Process Study", 15th International Conference on Design Science Research in Information Systems and Technology, Kristiansand, Norway, 2020, pp. 126-141.

[14] R. Meyer von Wolff, S. Hobert, K. Masuch, and M. Schumann, "Chatbots at Digital Workplaces - A Grounded-Theory Approach for Surveying Application Areas and Objectives", Pacific Asia Journal of the 
Association for Information Systems 12(2), 2020, pp. $63-$ 101.

[15] R. Winkler, E. Elshan, M. Söllner, and J. Leimeister, "SPAM - A Process Model for Developing Smart Personal Assistants", 53rd Hawaii International Conference on System Sciences, Maui, Hawaii, 2020, pp. 261-270.

[16] R.M. Schuetzler, G.M. Grimes, J.S. Giboney, and H.K. Rosser, "Deciding Whether and How to Deploy Chatbots", MIS Quarterly Executive 20(1), 2021, pp. 115.

[17] A.R. Hevner, S.T. March, J. Park, and S. Ram, "Design science in information systems research", MIS Quarterly 28(1), 2004, pp. 75-105.

[18] A.R. Hevner, "A Three Cycle View of Design Science Research", Scandinavian Journal of Information Systems 19(2), 2007, 1-6.

[19] A. Følstad and P.B. Brandtzæg, "Chatbots and the new world of HCI", interactions 24(4), 2017, pp. 38-42.

[20] S. Holmes, A. Moorhead, R. Bond, H. Zheng, V. Coates, and M. Mctear, "Usability testing of a healthcare chatbot: Can we use conventional methods to assess conversational user interfaces?", 31st European Conference on Cognitive Ergonomics, BELFAST United Kingdom, 2019, pp. 207-214.

[21] C. Corea, P. Delfmann, and S. Nagel, "Towards Intelligent Chatbots for Customer Care - Practice-Based Requirements for a Research Agenda", 53rd Hawaii International Conference on System Sciences, Maui, Hawaii, 2020, pp. 5819-5828.

[22] F. Johannsen, S. Leist, D. Konadl, and M. Basche, "Comparison of Commercial Chatbot Solutions for Supporting Customer Interaction", 26th European Conference on Information Systems, Portsmouth, UK, 2018, pp. 1-17.

[23] E. Elshan and P. Ebel, "Let's Team Up: Designing Conversational Agents as Teammates", 41th International Conference on Information Systems, India, 2020, pp. 1-9.

[24] R. Lechler, E. Stoeckli, R. Rietsche, and F. Uebernickel, "Looking Beneath the Tip of the Iceberg: The Two-Sided Nature of Chatbots and Their Roles for Digital Feedback Exchange", 27th European Conference on Information Systems, Sweden, 2019, pp. 1-17.

[25] N. Tavanapour, M. Poser, and E. Bittner, "Supporting the Idea Generation Process in Citizen Participation Toward an Interactive System with a Conversational Agent as a Facilitator", 27th European Conference on Information Systems, Sweden, 2019, pp. 1-17.

[26] S. Hobert, "Say Hello to 'Coding Tutor'! Design and Evaluation of a Chatbot-based Learning System Supporting Students to Learn to Program", 40th International Conference on Information Systems, Munich, Germany, 2019, pp. 1-17.

[27] C. Chakrabarti and G.F. Luger, "Artificial conversations for customer service chatter bots: Architecture, algorithms, and evaluation metrics", Expert Systems with Applications 42(20), 2015, pp. 6878-6897.

[28] S. Diederich, A.B. Brendel, and L.M. Kolbe, "Designing Anthropomorphic Enterprise Conversational Agents",
Business \& Information Systems Engineering 62(3), 2020, pp. 193-209.

[29] T. Rietz, I. Benke, and A. Maedche, "The Impact of Anthropomorphic and Functional Chatbot Design Features in Enterprise Collaboration Systems on User Acceptance", 14th International Conference on Wirtschaftsinformatik, Germany, 2019, pp. 1642-1656.

[30] R. Bavaresco, D. Silveira, E. Reis, J. Barbosa, R. Righi, C. Costa, R. Antunes, M. Gomes, C. Gatti, M. Vanzin, S.C. Junior, E. Silva, and C. Moreira, "Conversational agents in business: A systematic literature review and future research directions", Computer Science Review 36(4), 2020.

[31] S. Stieglitz, F. Brachten, and T. Kissmer, "Defining Bots in an Enterprise Context", 39th International Conference on Information Systems, San Francisco, USA, 2018, pp. 1-9.

[32] T. Lewandowski, J. Delling, C. Grotherr, and T. Böhmann, "State-of-the-Art Analysis of Adopting AIbased Conversational Agents in Organizations: A Systematic Literature Review", 25th Pacific Asia Conference on Information Systems, Dubai, UAE, 2021, pp. 1-14.

[33] A. Janssen, J. Passlick, D. Rodríguez Cardona, and M.H. Breitner, "Virtual Assistance in Any Context", Business \& Information Systems Engineering 62(3), 2020, pp. 211-225.

[34] M.T.P. Adam, S. Gregor, A. Hevner, and S. Morana, "Design Science Research Modes in Human-Computer Interaction Projects", AIS Transactions on HumanComputer Interaction 13(1), 2021, pp. 1-11.

[35] J. Feine, S. Morana, and A. Maedche, "Leveraging Machine-Executable Descriptive Knowledge in Design Science Research - The Case of Designing SociallyAdaptive Chatbots", International Conference on Design Science Research in Information Systems and Technology, Worcester, MA, USA, 2019, pp. 76-91.

[36] S. Hobert, "How Are You, Chatbot? Evaluating Chatbots in Educational Settings - Results of a Literature Review", 17. Fachtagung Bildungstechnologien (DELFI 2019), Bonn, 2019, pp. 259-270.

[37] https://conversations2020.wordpress.com/.

[38] J. Manseau, "AI in the Workplace: A Qualitative Analysis of Intelligent Employee Assistants", 26th Americas Conference on Information Systems, 2020, pp. 1-10.

[39] https://chatbots.org/.

[40] J. Feine, U. Gnewuch, S. Morana, and A. Maedche, "A Taxonomy of Social Cues for Conversational Agents", International Journal of Human-Computer Studies 132, 2019, pp. 138-161.

[41] R. Meyer von Wolff, T. Heuzeroth, S. Hobert, and M. Schumann, "The Students' View on IT-Support Chatbots at Universities - A Case-based Pilot Study", 26th Americas Conference on Information Systems, 2020, pp. 1-10.

[42] W. Maroengsit, T. Piyakulpinyo, K. Phonyiam, S. Pongnumkul, P. Chaovalit, and T. Theeramunkong, "A Survey on Evaluation Methods for Chatbots", 7th International Conference on Information and Education Technology, Japan, 2019, pp. 111-119. 